\title{
Enhancing the BER and ACLR for the HPA using pre-distortion technique
}

\author{
Ehab Abdulrazzaq Hussein \\ Department of Electrical Engineering, Faculty of Engineering, University of Babylon, Iraq
}

\begin{tabular}{l} 
Article Info \\
\hline Article history: \\
Received Mar 9, 2018 \\
Revised Jan 26, 2019 \\
Accepted Mar 11, 2019 \\
\hline
\end{tabular}

Keywords:

ACLR

BER

High power amplifier

Linearization technique

OFDM

Predistortion

\begin{abstract}
Power amplifiers are key components in wireless transceivers. Their function is to amplify signal and generate the required Radio Frequency (RF) power that allows to transmit the signal over an appropriate range. The Orthogonal Frequency Division Multiplexing (OFDM) systems are highly sensitive to nonlinear distortion introduced by High Power Amplifier (HPA). The HPA nonlinearity causes in-band and out-of-band distortions. The linearization techniques are used to compensate the nonlinear effects of the high power amplifier. These techniques correct the distortion effects resulting from nonlinearities in the transmitted signal. Many linearization techniques have been developed to improve power amplifier linearity and to decrease both Bit Error Rate (BER) and Adjacent Channel Leakage Ratio (ACLR). This work is set to run the high power amplifier in the nonlinear region. It is also attempting to analyze the resulting signal in terms of the BER and ACLR, next employs pre-distortion linearization techniques to reduce the distortion introduced in this region. According to Digital Video BroadcastingTerrestrial (DVB-T) standard the linearization techniques, circuit and the OFDM transmitter and receiver is designed and implemented through using computer simulation of AWR Design Environment.
\end{abstract}

Copyright () 2019 Institute of Advanced Engineering and Science. All rights reserved.

\section{Corresponding Author:}

Ehab Abdulrazzaq Hussein,

Department of Electrical Engineering,

University of Babylon,

Hillah-Najaf Road, Elect. Dept., Faculty of Eng., University of Babylon, Babylon, Iraq.

Email: dr.ehab@itnet.uobabylon.edu.iq

\section{INTRODUCTION}

OFDM, the multi-carrier transmission technique, is an excellent method for high speed bidirectional wireless data communication [1]. It is very similar to the well-known technique of Frequency Division Multiplexing (FDM). It uses the same principles of FDM to allow multiple messages to be sent over a single radio channel. It offers a much more controlled manner, which is allowing an improved spectral efficiency [2]. An OFDM signal consists of a sum of subcarriers that is modulated by using Phase Shift Keying (PSK) or Quadrature Amplitude Modulation (QAM). Although OFDM provides good spectral efficiency, it produces a signal with a fluctuating envelope that generates Intermodulation Distortion (IMD) at the system's power amplifiers. Most of the IMD power appears as an interference between adjacent channels, which requires the use of linear high power amplifiers. Considering back-off power is the simplest way to improve the linearity of the power amplifier which corresponds to moving the operating point of the amplifier to the linear region. Increasing the back-off of the power amplifier means that the signal is covered better in the linear range, and thus the effects of nonlinearities are reduced. However, power efficiency is reduced as well. So a tradeoff between efficiency and linearity is needed. Linearization techniques prove to be the best solution in order to improve power amplifier linearity without having a negative impact on efficiency [3]. Several kinds of linearization techniques for power amplifiers have been proposed such as 
Feedforward, Feedback, Predistortion, Linear Amplification using the Nonlinear Component [LINC] and Envelope Elimination and Restoration [EER]. This work aims to study the impact of amplifier nonlinearity for different modulation techniques and the effect of the pre-distortion linearization technique on it in OFDM system. Pre-distortion linearization technique is used widely because of its stability and its high efficiency.

\section{BASIC PRINCIPLE OF OFDM}

The OFDM idea utilizes information spreading which is transmitted over an extended number of carriers, each being balanced at a low rate. The transporters are generated to be orthogonal to each other by suitably picking the recurrence separating between them [5,6]. With a specific end goal to avoid countless channels on the transmitter and reciprocal channels and demodulators at the beneficiary, it is attractive to have the capacity to utilize the current computerized signal handling procedures, for example, Fast Fourier Transform (FFT) [7]. In a run of the mill OFDM transmission framework an information bit stream is injected into a channel encoder that partitions the information into $\mathrm{N}$ distinctive sub channels. The information is mapped onto M-PSK or M-QAM body. At that point, a N-point is connected onto OFDM mediator where IFFT is added to change the images from a recurrence area in the time space. Therefore, the time domain signals pass from parallel to serial transformation, and prepared to send. Today, the OFDM is commonly used for the most part in the transmission of computerized information where right now utilized on DAB, DVB, HDTV, ADSL, VDSL, Wireless LAN Networks, HIPERLAN/2, IEEE 802.11a, IEEE 802.11g, and IEEE 802.16 Broadband Wireless Access System.

Numerous advantages are gained by using OFDM, including: high range productivity as in Figure 1, strength to channel blurring, insensitivity to test timing balances than single bearer frameworks, invulnerability to motivation impedance, and simplicity of sifting through the clamor. The fundamental burden of OFDM framework can be described as follows: (a) its RF signal experiences a high crest to the normal force proportion (PAPR) because of the transmission of numerous sub-transporters and (b) it is very delicate to Inter Carrier Interference (ICI) because of recurrence counterbalance. The PAPR of a sign is given as:

$$
P A P R=\frac{\text { Signal Peak Value }}{\text { Signal Average value }}
$$

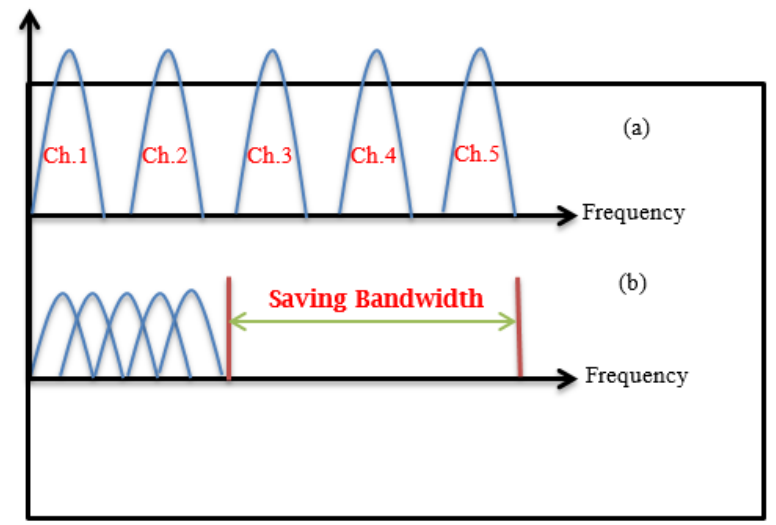

Figure 1. The concept of OFDM: (a) Multi-Carrier technique.

(b) Orthogonal Multi-Carrier technique.

\section{RESULTS AND ANALYSIS}

The force enhancer is the key component in the transmitter. In light of the vital issue, high power, productivity is a fundamental necessity for a powerful speaker. In the meantime, high linearity is increasingly alluring today, to minimize the recurrence obstruction and permit higher transmission limit in wideband correspondence frameworks. More client channels can be fitted into the accessible range if the range of linearity is extended in transmitter [8]. Nonlinear intensification yields IMD items and results in unsuitable ghastly regrowth in the contiguous channels. Direct intensification is required when the signal contains both 
sufficiency and state regulation. It can be proficient either by a chain of direct PAs or by a blend of nonlinear PAs [10]. In a perfect Power enhancer the unpredictable exchange capacity is given as:

$$
G=A e^{j \Phi}
$$

Where $\mathrm{G}$ and $\Phi$ is the linear gain and phase constant respectively. When a real amplifier is considered, the gain $\mathrm{A}(\mathrm{s}(\mathrm{t}))$ and phase-shift $\Phi(\mathrm{s}(\mathrm{t}))$ are represented as functions of the input signal $\mathrm{s}(\mathrm{t})$ while the complex transfer function depends on input power of the amplifier and is described mathematically as follows [5]:

$$
G(s(t))=A(s(t)) e^{j \Phi(s(t))}
$$

Two types of distortion are introduced due to the nonlinear characteristics of power amplifiers AM/AM (amplitude modulation/amplitude modulation) and AM/PM (amplitude modulation/phase modulation) effects. The characteristics of amplitude and phase of the output signal are known as AM-AM and AM-PM characteristics respectively. They result in output signal amplitude and phase modulation when the input signal envelope fluctuates [11]. The AM-AM and AM-PM characteristics can be obtained in digital communication systems as a function of the complex form of the input signal sample. For an $\mathrm{n}^{\text {th }}$ complex input sample $z_{n}$ with a magnitude $\left|z_{n}\right|$ and a phase are $\left(z_{n}\right)$, the complex transfer function of the nonlinear $\mathrm{PA}$ is represented by [5]:

$$
A\left(z_{n}\right)=A M\left(\left|z_{n}\right|^{2}\right) e^{j\left(\arg \left(z_{n}\right)+P M\left(\left|z_{n}\right|^{2}\right)\right)}
$$

Where $A M\left(\left|z_{n}\right|^{2}\right)$ and $P M\left(\left|z_{n}\right|^{2}\right)$ are mathematically modeled by polynomial functions, both of them are derived from the AM-AM and AM-PM characteristics, respectively. $\left|z_{n}\right|^{2}$ is the power of the $n^{\text {th }}$ complex input sample. Different factors can measure nonlinearity of the power amplifier such as third order intercept point (IP3), harmonics 1dB compression point, and Adjacent Channel Power Ratio (ACPR). The amount of signal energy which is leaking from the main an adjacent channel can be determined by the help of ACPR. The overall system response of non-constant modulated signals are expected to be enhanced using RF power amplifier linearization techniques. This can be achieved by reducing ACPR in the PA output power spectral density [3] as presented in the following equation:

$$
A C P R=\frac{\text { Total power in adjacent channel }}{\text { Power in the main channel }}
$$

\section{PROPOSED MODEL OF DISTORTION TECHNIQUE}

Proficiency is an essential sympathy toward the power amplifier creators. In any case, the tradeoff for high effectiveness is regularly results in reducing in linearity, and the diminishing linearity is added to ghastly obstruction outside the proposed transfer speed. Pre-distortion gives better effectiveness, lower expense and appropriateness of linearity from the other linearization systems. This is achieved based on the grounds that a nonlinear circuit is embedded between the information signal and the PA. This nonlinear circuit creates the IMD items backwards to those delivered by the PA and cross out the impact of PA nonlinearity accordingly. Figure 2 demonstrates an outline of a proposed pre-distortion linearizer.

The pre-distortion linearizer circuit is composed of two circles and three ways. Where the information signal is separated into three parallel ways in the circuit and afterward recombined toward the end of pre-distortion linearizer. Two ways are direct and can essentially be a length of transmission line. The other way is nonlinear trademark that can be acquired from a speaker crashed into immersion area.

In the first loop, the power amplifier amplifies the input signal in the upper branch with a voltage gain of $A_{v}$ which leads to amplify the input signal as well as the appearance of distortion voltage $v_{d}$ by a certain amount.

$$
v_{s}=v_{\text {in }} A_{v}+v_{d}
$$

The attenuator circuit with the transfer function $\frac{1}{A_{\mathrm{v}}}$ that located after the amplifier is used to decrease the amplified signal to the level of linear signal.

$$
v_{A}=\frac{v_{s}}{A_{v}}=v_{i n}+\frac{v_{d}}{A_{v}}
$$




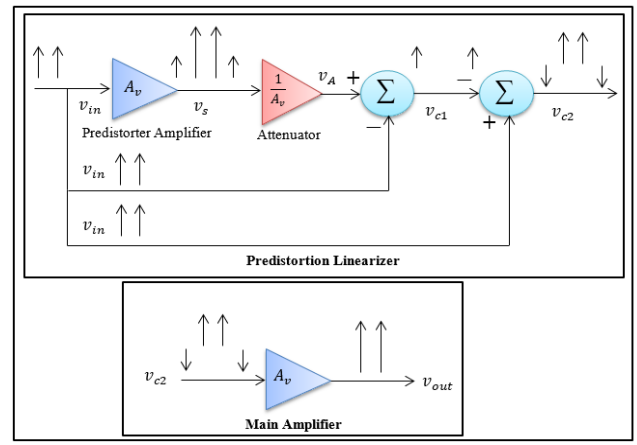

Figure 2. Block diagram of power amplifier system with predistortion linearizer.

The signal of the middle branch is a phase shifted version of the original RF signal. While the signal in the upper branch will be $180^{\circ}$ out of phase. This signal is contaminated with distortion that generated by the power amplifier. Thus, when the two signals in this loop are recombined at the output, will get only over the distortion signal.

$$
v_{c 1}=v_{A}-v_{i n}=v_{i n}+\frac{v_{d}}{A_{v}}-v_{i n}=\frac{v_{d}}{A_{v}} .
$$

In the second loop, the signal results from the first combiner is phase shifted by $180^{\circ}$ and recombined with the signal that coming from lower path. The result is the original signal minus it the output distortion that generated by the pre-distorter Amplifier.

$$
v_{c 2}=v_{i n}-v_{c 1}=v_{i n}-\frac{v_{d}}{A_{v}}
$$

The output signal that is coming from a circuit of pre-distortion linearize contains distortion which is the opposite of the distortion that results from amplification. They will cancel each other and the output signal will be almost free of distortion.

$$
\begin{gathered}
v_{\text {out }}=v_{\text {in }} A_{v}+v_{d} \\
v_{\text {out }}=\left(v_{\text {in }}-\frac{v_{d}}{A_{v}}\right) A_{v}+v_{d}=v_{\text {in }} A_{v}-v_{d}+v_{d} \\
v_{\text {out }}=v_{\text {in }} A_{v}
\end{gathered}
$$

The key to good predistortion filtering is to match the AM/AM and AM/PM responses of the distortion generating element with the AM/AM and AM/PM responses of the amplifier being pre-distorted.

\section{EXPERIMENTAL RESULTS}

Experimental work was carried out in this study using AWR Design Environment. The predistortion linearization technique is simulated for power amplifier in OFDM system.

\subsection{System parameters}

The OFDM signal for simulation parameters is selected based on the DVB-T standard as in Table 1.

Table 1. OFDM Model System Parameters

\begin{tabular}{ll}
\hline Source of Data & Random \\
\hline Type of Modulations & PSK, QAM \\
M & $16,64,256$ \\
Number of Subcarriers & 1705 \\
Power Amplifier & $\mathrm{G}=26, \mathrm{P} 1 \mathrm{~dB}=45$ \\
Coding & Convolution with rate $1 / 2$ \\
Mode & $2 \mathrm{k}$ \\
Subcarrier Spacing & $4.464(\mathrm{kHz})$ \\
Guard Interval & 0.03125 \\
Bandwidth & $7611.12(\mathrm{MHz})$ \\
\hline
\end{tabular}




\subsection{A Comparison of BER performances of 16PSK with 16QAM}

Figure 3 (a, b, and c) shows BER vs. SNR for 16PSK and 16QAM OFDM system with and without PDLT (Pre-Distortion Linearization Technique) for different values (15, 20 and 25) $\mathrm{dBm}$ of input power for OFDM system.

\subsection{A Comparison of BER performances of 64PSK with 64QAM} value of 64

Figure $4(a, b$, and c) shows the BER vs. SNR as in Figure 3 but the value of $M$ was changed to the

\subsection{A Comparison of BER performances of 256PSK with 256QAM}

BER performance results comparison with SNR for the OFDM system with 256PSK and 256QAM modulations with and without PDLT as shown in Figure 5 ( $a, b$, and $c)$.

Figures (3, 4 and 5) show BER vs. SNR for $(16,64,256)$ PSK and QAM, OFDM system with and without PDLT (Pre-Distortion Linearization Technique) for different values (15, 20 and 25) dBm of input power for OFDM system.

From the above shapes and tables, it can be observed that as the value of input power increased, the BER increases, where the input power impacts significantly on BER. Also, as SNR increased the PSK or QAM BER curve leans downward this refers to the reduction in bit error rate. Also, it is noticed that the value of the BER suffers significantly from reduction if used PDLT circuit.

\subsection{Power amplifier spectra}

Figure 6 ( $\mathrm{a}, \mathrm{b}, \mathrm{c}$ and d) presents the ACLR (Adjacent Channel Leakage Ratio) level of the power amplifier with and without the presence of pre-distortion linearization technique and different values of the input power level.

From Figure 6 (a, b, c and d) it is noticed that the spectral regrowth of amplifier reduced when used predistortion technique, which will lead to the reduction of the ACLR of the power amplifier.

\subsection{AM to AM of power amplifier}

Figure 7 displays AM to AM of predistortion linearizer and PA with and without using of predistortion linearization technique. From Figure 7, it can be observed that the linear region of the power amplifier, is increased significantly when we use PDLT because the transfer function of the predistortion linearizer is the opposite of the transfer function of PA in the nonlinear region.

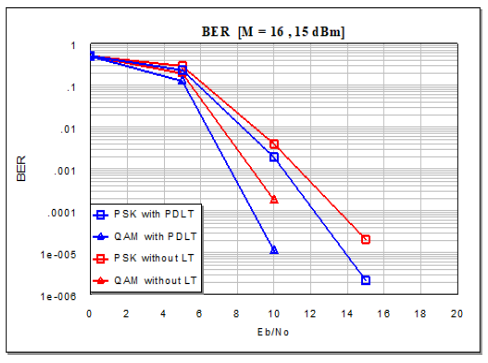

(a)

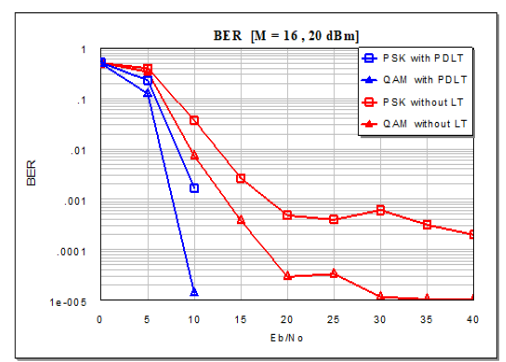

(b)

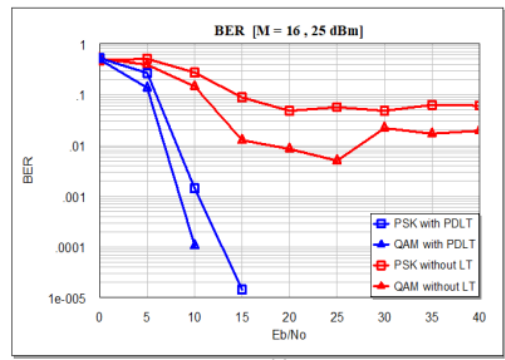

(c)

Figure 3 ( $\mathrm{a}, \mathrm{b}$ and $\mathrm{c})$. BER performance of 16PSK \& 16QAM in OFDM system with and without PDLT

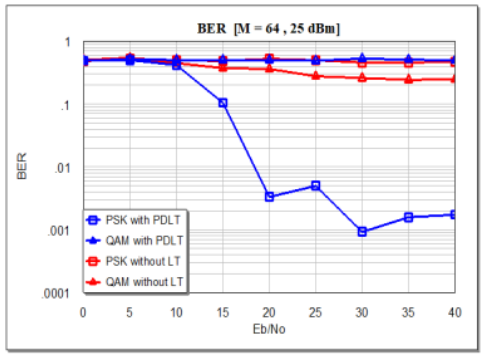

(a)

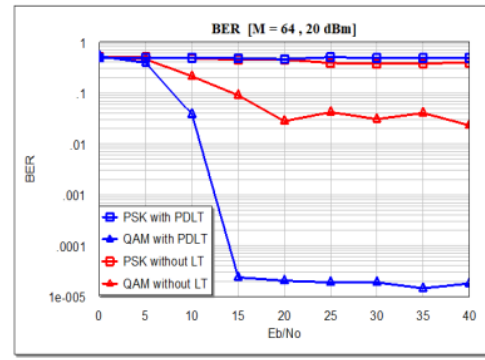

(b)

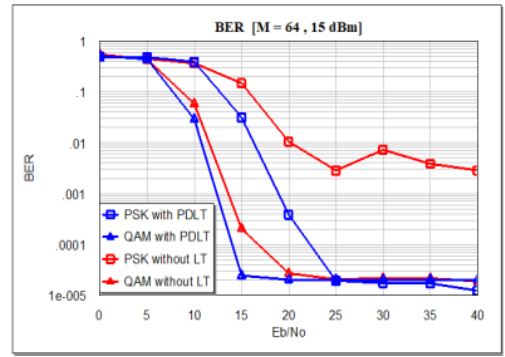

(c)

Figure 4 (a, b and c). BER performance of 64PSK \& 64QAM in OFDM system with and without PDLT 


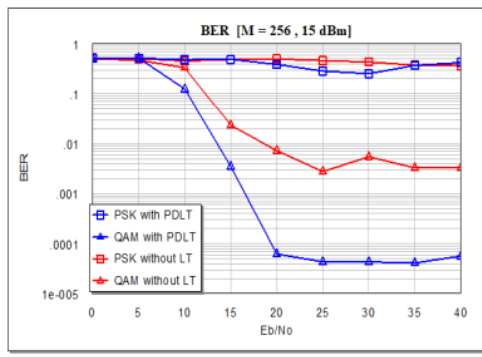

(a)

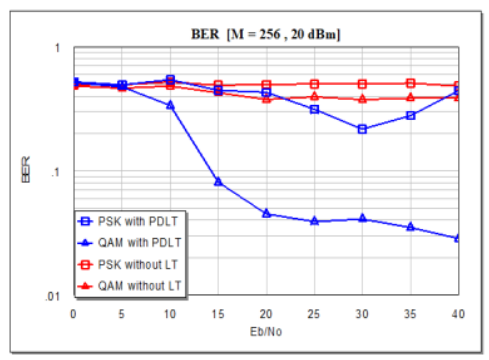

(b)

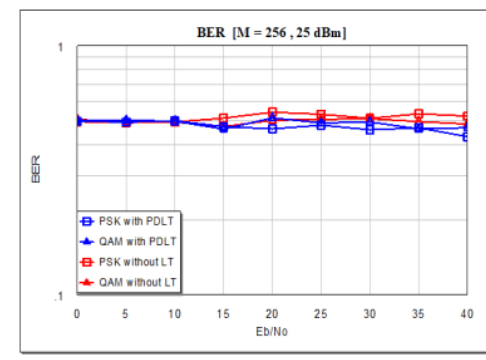

(c)

Figure 5 ( $\mathrm{a}, \mathrm{b}$ and $\mathrm{c}$ ). BER performance of 256PSK \& 256QAM in OFDM system with and without PDLT

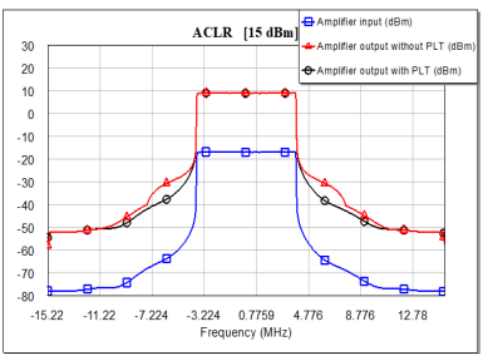

(a)

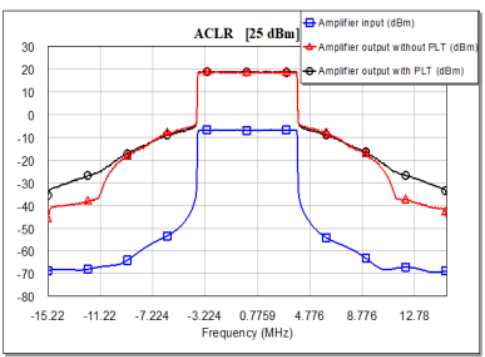

(c)

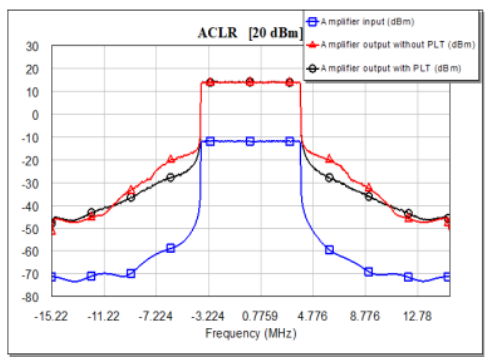

(b)

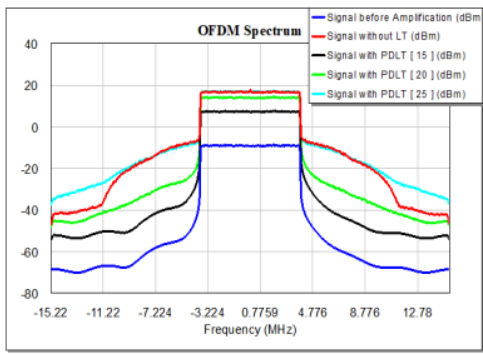

(d)

Figure 6 (a, b, c and d). Power amplifier spectra

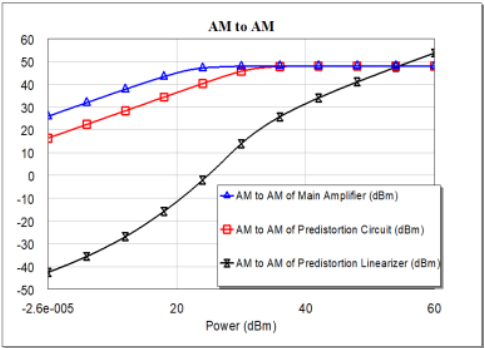

Figure 7. AM to AM of Power Amplifier

\section{CONCLUSIONS}

This work has developed a technique to run the high power amplifier in the nonlinear region. The results depict following conclusions: It is noticed that when using the pre-distortion method can be reduced the nonlinearity of the HPA. This can be enhanced BER and ACLR which will increase the efficiency of the HPA, and that will lead to an increase in the efficiency of OFDM system. It is also noticed that a bit error rate of the power amplifier depends on the types of modulation methods and Input Power (IP). Thus, it is less for the signal modulated with QAM as compared to PSK modulation and whenever the value of the IP has been reduced the results will be better. So the signal which is modulated with QAM has minimum distortion in OFDM system with the least possible value of IP. 


\section{REFERENCES}

[1] Paloma García-Dúcar, Jesús de Mingo and Antonio Valdovinos, "Misalignments Feedforward Transmitter Correction Design for Nonlinear Distortion Cancellation in OFDM Systems," 1-4244-0063-i2006 IEEE, 2006.

[2] A. Singh, H. Kaur, "Nonlinearity Analysis of High Power Amplifier in OFDM System," International Journal of Computer Applications, Vol. 37- No.2, January 2012.

[3] Ziad El-Khatib, Leonard MacEachern and Samy A. Mahmoud, "Distributed CMOS Bidirectional Amplifiers," Carleton University, 2012.

[4] Suranjana Julius, "Design and Implementation of An ETSI-SDR OFDM Transmitter with Power Amplifier Linearization," M.Sc. Thesis, University of Saskatchewan, 2010.

[5] Ehab A. Hussein, Mohammed A. Abdulkadhim, "Performance Improvement of BER in OFDM System using Feed Forward Technique on Power Amplifier," International Journal of Computer Applications (IJCA), Vol. 75, No.4, August 2013.

[6] Brahmaji T.A.R.K, “An efficient ICI Cancellation Technique for OFDM Communication Systems,” M.Sc. Thesis. National Institute of Technology, Rourkela, 2009.

[7] Ming Xiao, "Novel Predistortion Techniques for RF Power Amplifiers", The University of Birmingham, 2009.

[8] Frederick H. Raab, Peter Asbeck, Steve Cripps, Peter B. Kenington, Zoya B. Popovic, Nick Pothecary, John F. Sevic and Nathan O. Sokal, "RF and Microwave Power Amplifier and Transmitter Technologies — Part 1," High Frequency Electronics, May 2003.

[9] Tzi-Dar Chiueh and Pei-Yun Tsai, "OFDM Baseband Receiver Design for Wireless Communications," National Taiwan University, Taiwan, 2007.

\section{BIOGRAPHY OF AUTHOR}

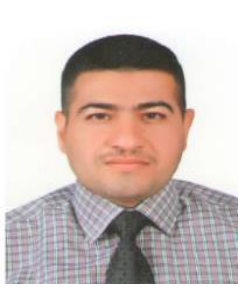

Ehab Abdulrazzaq Hussein, PhD. MSc. Electrical Engineering was born in Babylon on January 1, 1976. He obtained his BSc degree (1997) in Electrical Engineering at the Faculty of Engineering, University of Babylon and MSc degree (2000), in electrical engineering at the Department of Electrical Engineering, University of Technology and his PhD. Degree from the Department of Electrical Engineering at the Faculty of Engineering, University of Basrah, Currently he works as assistant professor at the Electrical Department at the Faculty of Engineering, University of Babylon. His main interest is signal processing, analysis, information transition, sensors and control system analysis. 